\title{
Evaluating Functional Annotations of Enzymes Using the Gene Ontology
}

\author{
Gemma L. Holliday, Rebecca Davidson, Eyal Akiva, and Patricia C. Babbitt
}

\begin{abstract}
The Gene Ontology (GO) (Ashburner et al., Nat Genet 25(1):25-29, 2000) is a powerful tool in the informatics arsenal of methods for evaluating annotations in a protein dataset. From identifying the nearest well annotated homologue of a protein of interest to predicting where misannotation has occurred to knowing how confident you can be in the annotations assigned to those proteins is critical. In this chapter we explore what makes an enzyme unique and how we can use GO to infer aspects of protein function based on sequence similarity. These can range from identification of misannotation or other errors in a predicted function to accurate function prediction for an enzyme of entirely unknown function. Although GO annotation applies to any gene products, we focus here a describing our approach for hierarchical classification of enzymes in the Structure-Function Linkage Database (SFLD) (Akiva et al., Nucleic Acids Res 42(Database issue):D521-530, 2014) as a guide for informed utilisation of annotation transfer based on GO terms.
\end{abstract}

Key words Catalytic function, Enzyme, Misannotation, Evidence of function

\section{Introduction}

Enzymes are the biological toolkit that organisms use to perform the chemistry of life, and the Gene Ontology (GO) [1] represents a detailed vocabulary of annotations that captures many of the functional nuances of these proteins. However, the relative lack of experimentally validated annotations means that the vast majority of functional annotations are electronically transferred, which can lead to erroneous assumptions and missannotations. Thus, it is important to be able to critically examine functional annotations. This chapter describes some of the key concepts that are unique for applying GO-assisted annotation to enzymes. In particular we introduce several techniques to assess their functional annotation within the framework of evolutionarily related proteins (superfamilies). 


\subsection{Enzyme Nomenclature and How It Is Used in GO}

At its very simplest, an enzyme is a protein that can perform at least one overall chemical transformation (the function of the enzyme). The overall chemical transformation is often described by the Enzyme Commission (EC) Number [2-4] (and see Chap. 19 [5]). The EC Number takes the form A.B.C.D, where each position in the code is a number. The first number (which ranges from 1 to 6) describes the general class of enzyme, the second two numbers (which both range from 1 to 99) describe the chemical changes occurring in more detail (the exact meaning of the numbers depends on the specific class of enzyme you are looking at) and the final number (formally ranging from 1 to 999) essentially describes the substrate specificity. The EC number has many limitations, not least the fact that it doesn't describe the mechanism (the manner in which the enzyme performs its overall reaction) and often contains no information on cofactors, regulators, etc. Nor is it structurally contextual [6] in that similarity in EC number does not necessarily infer similarity in sequence or structure, making it sometimes risky to use for annotation transfer, especially among remote homologous proteins. However, it does do exactly what it says on the tin: it defines the overall chemical transformation. This makes it an important and powerful tool for many applications that require a description of enzyme chemistry.

The Molecular Function Ontology (MFO) in GO contains the full definition of around $70 \%$ of all currently available EC numbers. Theoretically, the MFO would contain all EC numbers available. However, due to many EC numbers not currently being assigned to a specific protein identifier within UniProtKB, the coverage is lower than might be expected. Another important difference between the EC hierarchy and the GO hierarchy is that the latter is often much more complex than the simple four steps found in the EC hierarchy. For example, the biotin synthase (EC 2.8.1.6) hierarchy is relatively simple and follows the four step nomenclature, while the GO hierarchy for [cytochrome c]-arginine N-methyltransferase (EC 2.1.1.124) is much more complex (see Fig. 1).

Formally, MFO terms describe the activities that occur at the molecular level; this includes the "catalytic activity" of enzymes or "binding activity". It is important to remember that EC numbers and MFO terms represent activities and not the entities (molecules, proteins or complexes) that perform them. Further, they do not specify where, when or in what context the action takes place. This is usually handled by the Cellular Component Ontology. The final ontology in GO, the Biological Process Ontology (BPO), provides terms to describe a series of events that are accomplished by one or more organised assemblies of molecular functions. Each MFO term describes a unique single function that means the same thing regardless of the evolutionary origin of the entity annotated with that term. Although the BPO describes a collection of activities, some BPO terms can be related 
a

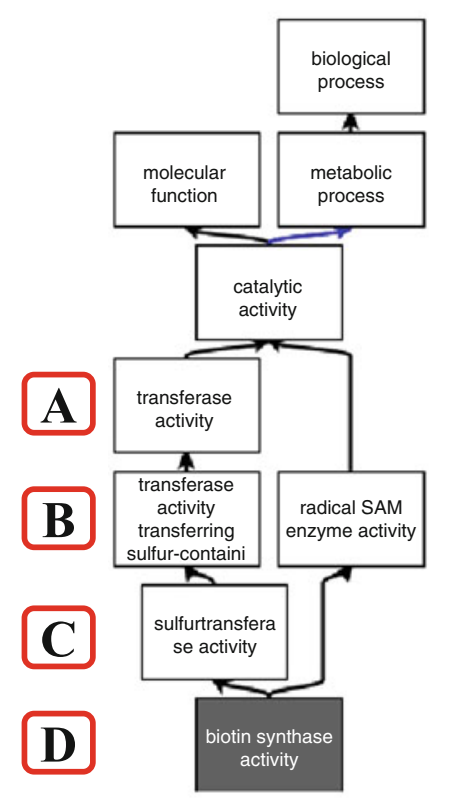

b

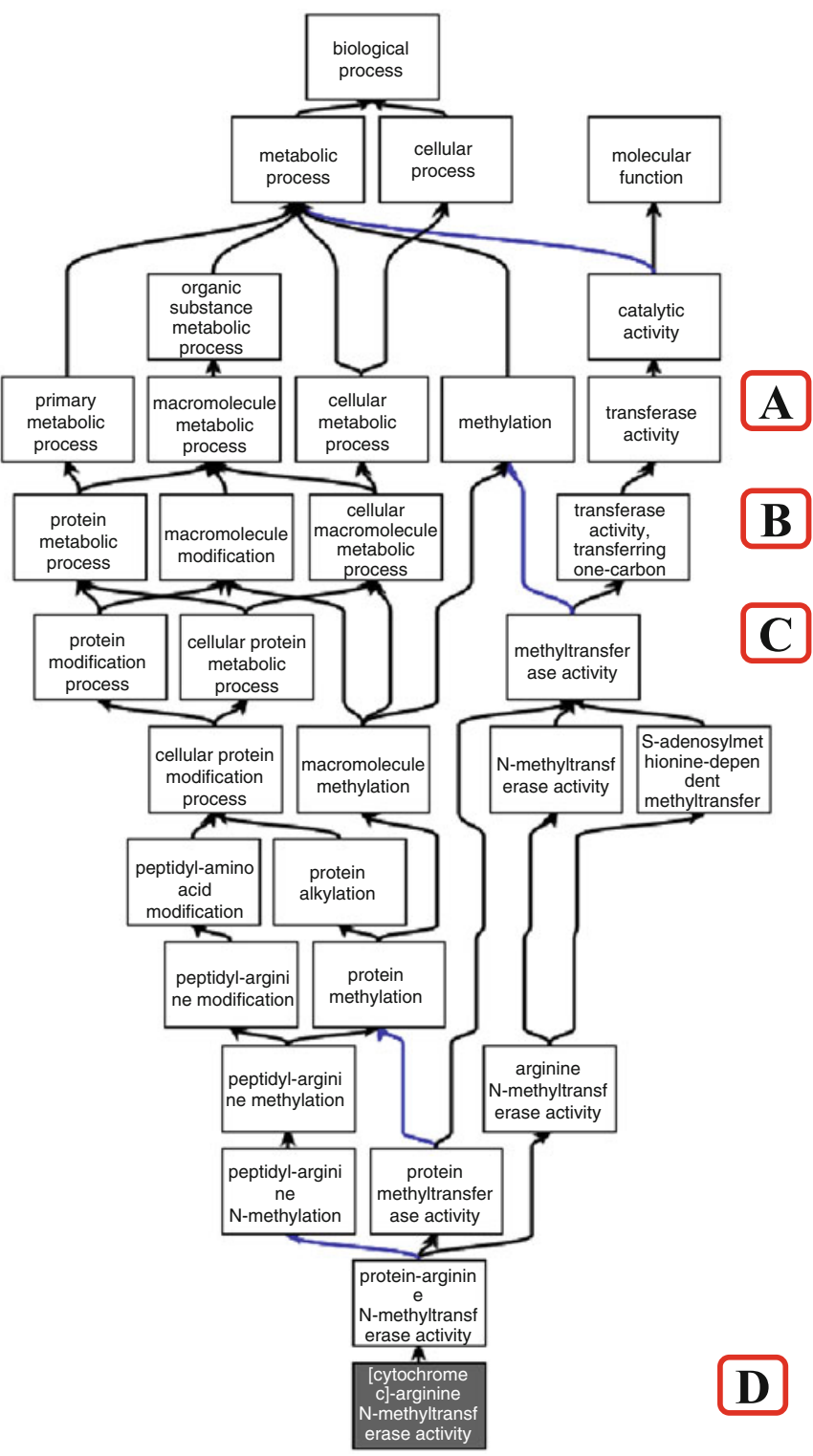

Fig. 1 Example of the GO hierarchy (taken from the ancestor chart of the QuickGo website (http://www.ebi. ac.uk/QuickGO/) showing the relative complexity of the GO hierarchy for two distinct EC numbers). (a) Shows the GO hierarchy for biotin synthase, EC 2.8.1.6; (b) shows the GO hierarchy for [cytochrome c]-arginine $\mathrm{N}$-methyltransferase, EC 2.1.1.24. The colours of the arrows in the ontology are denoted by the key in the centre of the figure. Black connections between terms represent an is_a relationship, blue connections represent a part_of relationship. The $A, B, C$ and $D$ in red boxes denote the four levels of the EC nomenclature

to their counterparts in the MFO, e.g. GO:0009102 (biotin biosynthetic process) could be considered to be subsumed with the MFO term GO:0004076 (biotin synthase activity) as GO:0009102 includes the activity GO:0004076, i.e. in such cases, the terms are interchangeable for the purpose of evaluation of a protein's 


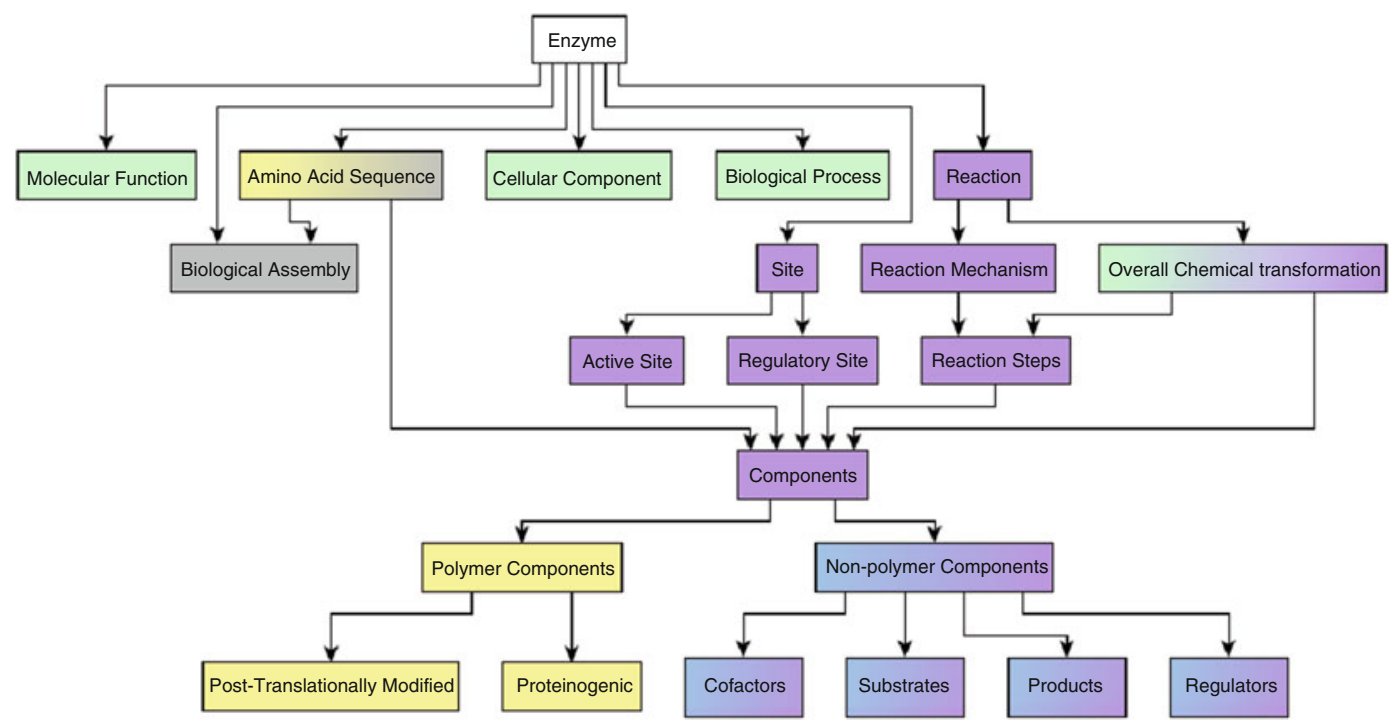

Fig. 2 Hierarchical view of enzyme features. The GO ontologies which describe proteins and their features are highlighted in light green. Other ontologies available in $\mathrm{OBO}$ and BioPortal are shown in the following colours: light yellow represents the Amino Acid Ontology, purple represents the Enzyme Mechanism Ontology, blue represents the ChEBI ontology and grey represents the Protein Ontology. See also Chap. 5 [10]. The terms immediately beneath the parent term are those terms that are covered by ontologies, and required for a protein to be considered an enzyme

annotation. Please see Chap. 2 [7] for a more in-depth discussion of the differences between BPO and MFO.

As a protein, an enzyme has many features that can be described and used to define the enzyme's function, from the primary amino acid sequence to the enzyme's quaternary structure (biological assembly), the chemistry that is catalysed, to the localisation of the enzyme. Features can also denote the presence (or absence) of active site residues to confirm (or deny) a predicted function, such as EC class, using the compositional makeup of a protein amino acid sequences $[8,9]$. Nevertheless, for the many proteins of unknown function deposited in genome projects, prediction of the molecular, biological, and cellular functions remains a daunting challenge. Figure 2 provides a view of enzyme-specific features along with the GO ontologies that can also be used to describe them. Because it captures these features through a systematic and hierarchical classification system, GO is heavily used as a standard for evaluation of function prediction methods. For example, a regular competition, the Critical Assessment of Functional Annotation (CAFA) has brought many in the function prediction community together to evaluate automated protein function prediction algorithms in assigning GO terms to protein sequences [11]. Please see Chap. 10 [12] for a more detailed discussion of CAFA. 


\subsection{Why Annotate Enzymes with the Gene Ontology?}

Although there are many different features and methods that can (and are) used to predict the function of a protein, there are several advantages to using GO as a broadly applied standard. Firstly, GO has good coverage of known and predicted functions so that nearly all proteins in GO will have at least one associated annotation. Secondly, annotations associated with a protein are accompanied by an evidence code, along with the information describing that evidence source. Within the SFLD [13] each annotation has an associated confidence level which is linked to both the evidence code, source of the evidence (including the type of experiment) and the curator's experience. For example, experimental evidence for an annotation is considered as having high confidence whereas predictions generated by computational methods are considered of lower confidence (Chap. 3 [14]). In general there are three types of evidence for the assignment of a GO term to a protein:

1. Fully manually curated: These proteins will usually have an associated experimental evidence that has been identified by human curators and who have added relevant evidence codes. For the purposes of the SFLD and this chapter, these are considered high confidence and will have a greater weight than any other annotation confidence level.

2. Computational with some curator input: These are computationally based annotations that have been propagated through curator derived rules, and are generally considered to be of medium confidence by the SFLD. Due to the huge proportion of sequences in large public databases now available, over $98 \%$ of GO annotations are inferred computationally [15].

3. Computational with no curator input: These annotations that have been computationally inferred from information without any curator input into the inference rules and are considered to be of the lowest confidence by the SFLD.

All computationally derived annotations rely upon prior knowledge, and so if the rule is not sufficiently detailed, it can still lead to the propagation of annotation errors (see Misannotation Section 1.4).

Assigning confidence to annotations is highly subjective [16], however, as one person may consider high-throughput screening, which more frequently is used to predict protein-binding or subcellular locations rather than EC number, of low confidence. This is because such experiments often have a relatively high number of false positives that can generate bias in the analysis. However, depending on what your research questions are, you may consider such data of high confidence. It all depends on what field you are in and what your needs are. Generally speaking, the more reproducible the experiment(s), the higher confidence you can have in their results. Thus, even low-to-medium confident annotations (from Table 1) may lead to a high-confidence annotation. 
Table 1

Some example proteins (listed by UniProtKB accession) with their associated annotations, source of the annotation (the SFLD is the Structure-Function Linkage Database, Swiss-Prot is the curated portion of UniProtKB) and the confidence of those annotations along with the reason that confidence level has been assigned

\begin{tabular}{|c|c|c|c|}
\hline $\begin{array}{l}\text { Protein ID from } \\
\text { UniProtKB [17] }\end{array}$ & $\begin{array}{l}\text { Annotated protein } \\
\text { function (source) }\end{array}$ & $\begin{array}{l}\text { SFLD } \\
\text { confidence } \\
\text { level }\end{array}$ & $\begin{array}{l}\text { Types of evidence or reasoning used to annotate } \\
\text { the function }\end{array}$ \\
\hline Q9X0Z6 & $\begin{array}{l}\text { [FeFe]-hydrogenase } \\
\text { maturase (From } \\
\text { SFLD and } \\
\text { Swiss-Prot) }\end{array}$ & High & $\begin{array}{l}\text { Inferred from experimental analysis of protein } \\
\text { structures, genomic context and results from } \\
\text { spectroscopic assay. }\end{array}$ \\
\hline Q11S94 & $\begin{array}{l}\text { Biotin Synthase } \\
\text { (BioB) (From } \\
\text { SFLD and } \\
\text { Swiss-Prot) }\end{array}$ & Medium & $\begin{array}{l}\text { Inferred from similarity to other BioB enzymes. } \\
\text { Matched by similarity to other BioB sequences } \\
\text { and catalytic residues are fully conserved. }\end{array}$ \\
\hline Q58692 & $\begin{array}{l}\text { Biotin Synthase } \\
\text { (BioB) (From } \\
\text { Swiss-Prot) }\end{array}$ & Low & $\begin{array}{l}\text { Inferred from similarity to other BioB enzymes. } \\
\text { Matched by similarity to other BioB sequences. } \\
\text { Whilst all residues required for binding the } \\
\text { iron-sulphur clusters are conserved, all the } \\
\text { catalytic residues (those required for the BioB } \\
\text { reaction to occur) are not. Also has no biotin } \\
\text { synthase genomic context. }\end{array}$ \\
\hline
\end{tabular}

1.3 Annotation Transfer Under the Superfamily Model
For example the GO Reference Code GO_REF:0000003 provides automatic GO annotations based on the mapping of EC numbers to MFO terms, so although annotated as IEA, these annotations can be considered of higher confidence [18]. Some examples of high-, medium- and low-confidence annotations are shown in Table 1, along with reference to the approach used in SwissProt and the SFLD to describe their reliability.

We define here an enzyme (or protein) superfamily as the largest grouping of enzymes for which a common ancestry can be identified. Superfamilies can be defined in many different ways, and every resource that utilises them in the bioinformatics community has probably used a slightly different interpretation and method to collate their data. However, they can be broadly classified as structurebased, in which the three-dimensional structures of all available proteins in a superfamily have been aligned and confirmed as homologous, or sequence based, where the sequences have been used rather than structures. Many resources use a combination of approaches. Examples of superfamily based resources include CATH [19], Gene3D [20], SCOP and SUPERFAMILY [21], which are primarily structure based, and Pfam [22], PANTHER [23] and TIGRFAMs [24], which are primarily sequence based. A third definition of a superfamily includes a mechanistic component, i.e. a set 
of sequences must not only be homologous, but there must be some level of conserved chemical capability within the set, e.g. catalytic residues, cofactors, substrate and/or product substructures or mechanistic steps. An example of such a resource is the SFLD and we will focus on this resource with respect to evaluating GO annotations for enzymes that are members of a defined superfamily.

The SFLD (http://sfld.rbvi.ucsf.edu/) is a manually curated classification resource describing structure-function relationships for functionally diverse enzyme superfamilies [25]. Members of such superfamilies are diverse in their overall reactions yet share a common ancestor and some conserved active site features associated with conserved functional attributes such as a partial reaction or molecular subgraph that all substrates or products may have in common. Thus, despite their different functions, members of these superfamilies often "look alike" which can make them particularly prone to misannotation. To address this complexity and enable reliable transfer of functional features to unknowns only for those members for which we have sufficient functional information, we subdivide superfamily members into subgroups using sequence information (and where available, structural information), and lastly into families, defined as sets of enzymes known to catalyse the same reaction using the same mechanistic strategy and catalytic machinery. At each level of the hierarchy, there are conserved chemical capabilities, which include one or more of the conserved key residues that are responsible for the catalysed function; the small molecule subgraph that all the substrates (or products) may include and any conserved partial reactions. A subgroup is essentially created by observing a similarity threshold at which all members of the subgroup have more in common with one another than they do with members of another subgroup. (Thresholds derived from similarity calculations can use many different metrics, such as simple database search programs like BLAST [26] or Hidden Markov Models (HMMs) [27] generated as part of the curation protocol to describe a subgroup or family.)

\subsection{Annotation Transfer and Misannotation}

Annotation transfer is a hard problem to solve, partly because it is not always easy to know exactly how a function should be transferred. Oftentimes, function and sequence similarity do not track well $[28,29]$ and so, if sequence similarity is the only criterion that has been used for annotation transfer, the inference of function may have low confidence. However, it is also very difficult to say whether a protein is truly misannotated, especially if no fairly similar protein has been experimentally characterised that could be used for comparison and evaluation of functional features such as the presence of similar functionally important active site residues. As we have previously shown [30-32] there is a truly staggering amount of protein space that has yet to be explored experimentally and that makes it very difficult to make definitive statements as to the validity of an annotation. 
Misannotation can come from many sources, from a human making an error in curation, which is then propagated from the top down, to an automated annotation transfer rule that is slightly too lax, to the use of transitivity to transfer annotation, e.g. where protein $\mathrm{A}$ is annotated with function $\mathrm{X}$, protein $\mathrm{B}$ is $70 \%$ identical to $\mathrm{A}$, and so is also assigned function $\mathrm{X}$, protein $\mathrm{C}$ is $65 \%$ identical to protein $\mathrm{B}$, and so is also assigned function $\mathrm{X}$. Whilst this may be the correct function, protein $\mathrm{C}$ may have a much lower similarity to protein A, and thus the annotation transfer may be "risky" [33]. As in the example shown in Fig. 3, sequence similarity networks (SSNs) [34] offer a powerful way to highlight where potential

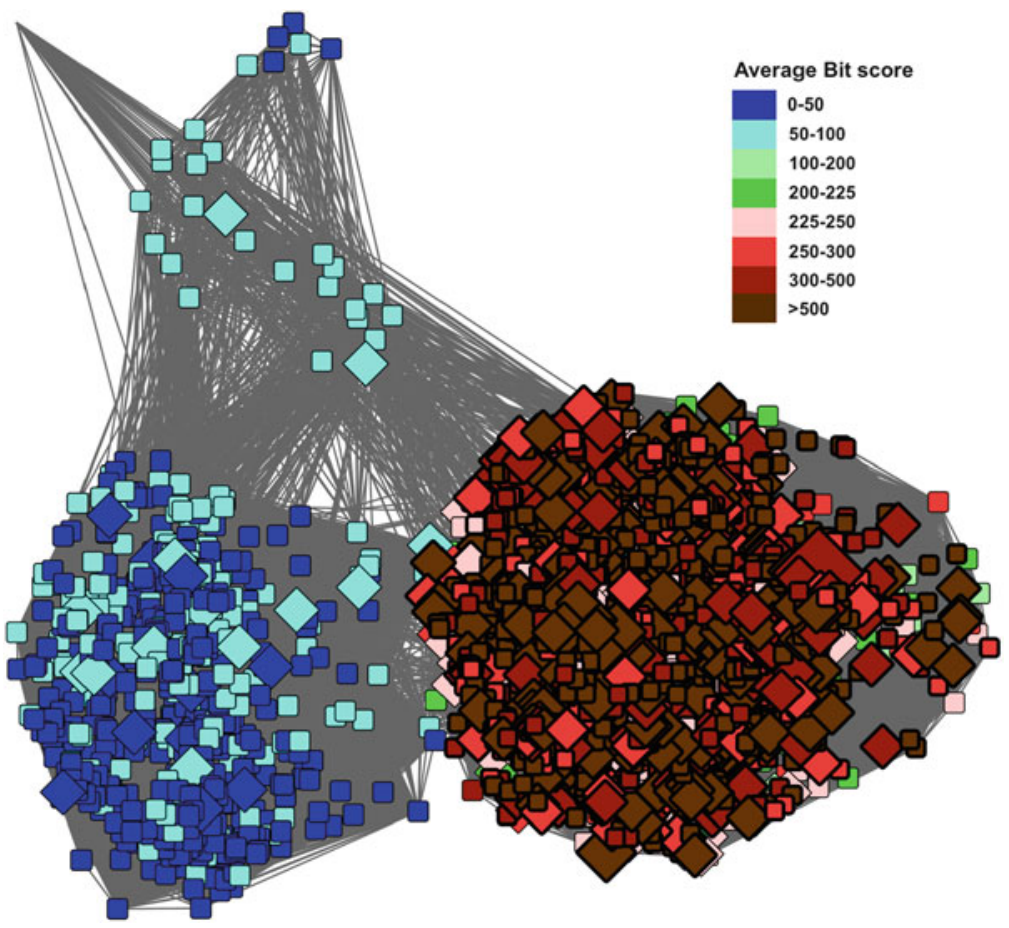

Fig. 3 Example of identifying misannotation using an SSN in the biotin synthaselike subgroup in the SFLD. Nodes colours represent different families in the subgroup, where red represent those sets of sequences annotated as canonical biotin synthase in the SFLD, blue represent the HydE sequences, green the PyIB sequences and magenta the $\mathrm{HmdB}$ sequences. The nodes shown as large diamonds are those annotated as BioB in G0, clearly showing that the annotation transfer for $\mathrm{BioB}$ is too broad. The network summarizes the similarity relationships between 5907 sequences. It consists of 2547 representative nodes (nodes represent proteins that share greater than $90 \%$ identity) and 2,133,749 edges, where an edge is the average similarity of pairwise BLAST E-values between all possible pairs of the sequences within the connected nodes. In this case, edges are included if this average is more significant than an $E$-value of $1 \mathrm{e}-25$. The organic layout in Cytoscape 3.2.1 is used for graphical depiction. Subheading 2.1 described how such similarity networks are created 
misannotation may occur. In this network, all the nodes are connected via a homologous domain, the Radical SAM domain. Thus, the observed differences in the rest of the protein mean that the functions of the proteins may also be quite different. For details on the creation of SSNs, see Subheading 2.1. Cases where annotations may be suspect can often be evaluated based on a protein's assigned name, and from the GO terms inferred for that protein.

Not all annotations are created equal, even amongst experimentally validated annotations, and it is important to consider how well evidence supporting an annotation should be trusted. For example, in the glutathione transferase (GST) superfamily, the cognate reaction is often not known as the assays performed use a relatively standard set on non-physiological substrates to infer the type of reaction catalysed by each enzyme that is studied. Moreover, GSTs are often highly promiscuous for two or more different reactions again complicating function assignment [32]. That being said, the availability of even a small amount of experimental evidence can help guide future experiments aimed at functional characterisation. A new ontology, the Confidence Information Ontology (CIO) [16], aims to help annotators assign confidence to evidence. For example, evidence that has been reproduced from many different experiments may have an intrinsically higher confidence than evidence that has only been reported once.

\section{Using GO Annotations to Visualise Data in Sequence Similarity Networks}

Sequence similarity networks (SSNs) are a key tool that we use in the Structure-Function Linkage Database (SFLD) as they give an immediately accessible view of the superfamily and the relationships between proteins in this set. This in turn allows a user to identify boundaries at which they might reasonably expect to see proteins performing a similar function in a similar manner. As was shown in Fig. 3, the GO annotation for BioB covered several different SFLD families. These annotation terms have been assigned through a variety of methods, but mostly inferred from electronic annotation (i.e. rule-based annotation transfer as shown in Fig. 4).

From the networks shown previously, a user may intuitively see that there are three basic groups of proteins. Further, it could be hypothesised that these groups could have different functions (which is indeed the case in this particular example). Thus, the user may be left with the question: How do I know what boundaries to use for high confidence in the annotation transfer? Figure 5 shows another network, this time coloured by the average bit-score for the sequences in a node against the SFLD HMM for BioB. This network exemplifies how (1) sequence similarity (network clusters) corresponds with the sequence pattern generated by SFLD curators to represent the BioB family, and (2) HMM true-positive gathering 


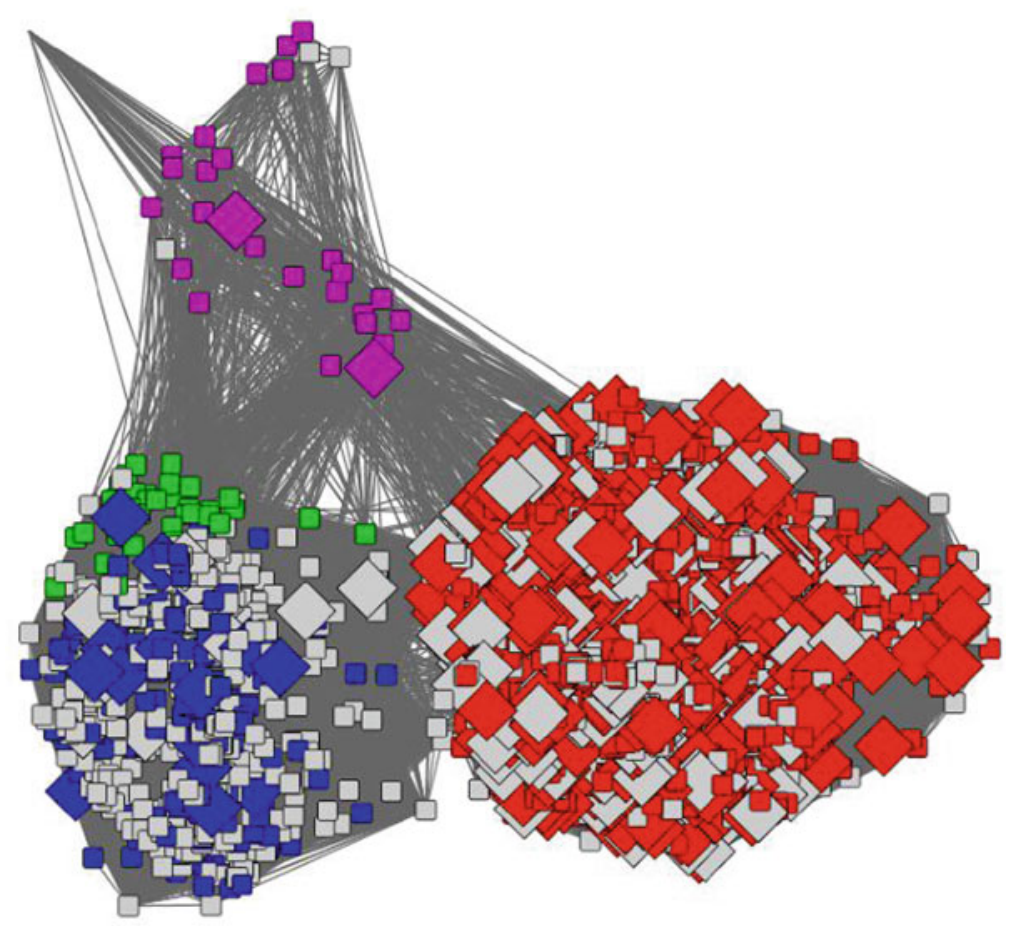

Fig. 4 Biotin synthase-like subgroup coloured by confidence of evidence (as shown in Table 1). The diamond shaped nodes are all annotated as Biotin Synthase in G0. Red nodes are those that only have low confidence annotations, the orange nodes are those that have at least one medium-confidence annotations and the green are those that have at least one high-confidence annotation. Grey nodes have no BioB annotations. Node and edge numbers, as well as $e$-value threshold are as in Fig. 3

bit-score cut-off can be fine-tuned. By combining what we know about the protein set from the GO annotation (Fig. 3) with the HMM bit-score (Fig. 5) it is possible to be much more confident in the annotations for the proteins in the red/brown group in Fig. 5.

\subsection{Creating}

Sequence Similarity Networks
SSNs provide a visually intuitive method for viewing large sets of similarities between proteins [34]. Although their generation is subject to size limitations for truly large data sets, they can be easily created and visualised for several thousand sequences. There are many ways to create such networks, the networks created by the SFLD are generated by Pythoscape [35], a freely available software that can be downloaded, installed and can be run locally. Recently, web servers have been described that will generate networks for users. For example, The Enzyme Similarity Tool (EFI-EST) [36] created by the Enzyme Function Initiative will take a known set of proteins (e.g. Pfam or InterPro [37] groups) and generate networks for users from that set. A similarity network is simply a set of nodes (representing a set of amino acid sequences as described in 


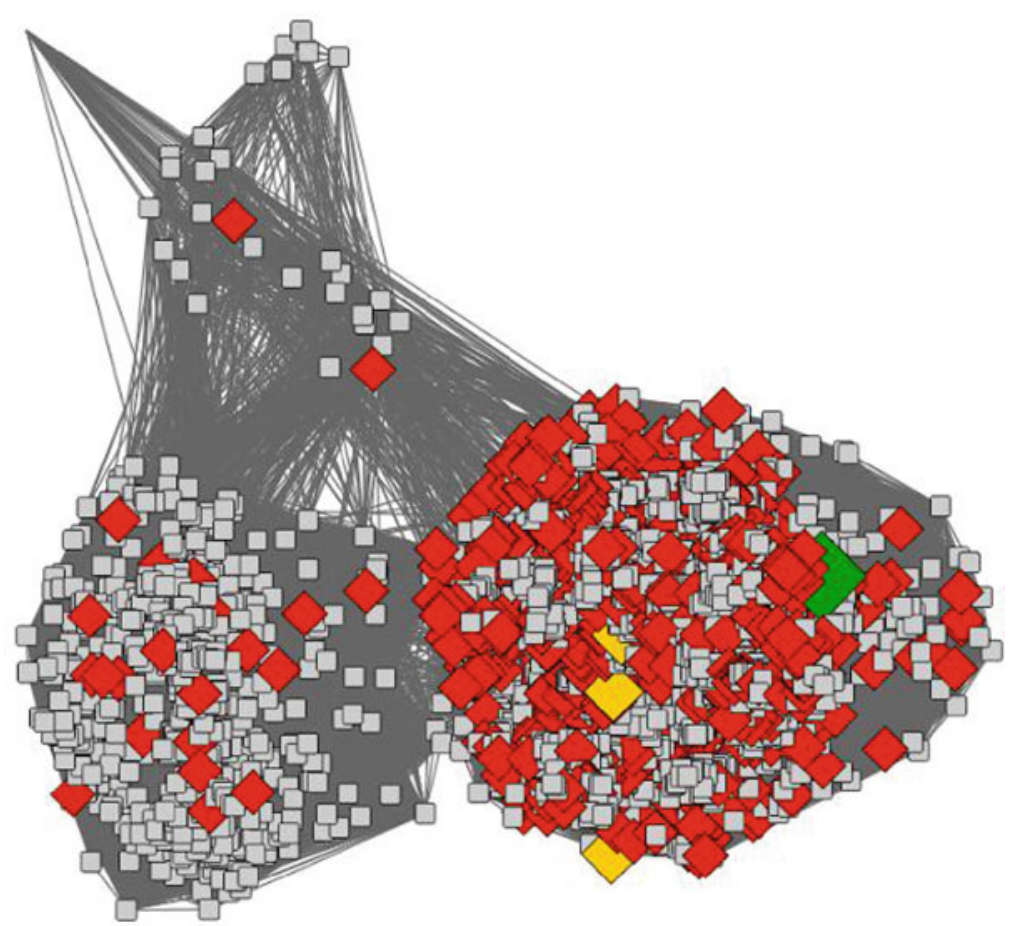

Fig. 5 Example of a sequence similarity network to estimate subgroups for use in initial steps of the curation process and to guide fine-tuning the hidden Markov model (HMM) true-positive detection threshold of an enzyme family (here for the Biotin synthase (BioB) family). Node colours represent the average Bit-score of the BioB family HMM for all sequences represented by the node. The mapping between colours and average Bit scores is given in the legend. Nodes with thick borders represent proteins that belong to the BioB family according to SFLD annotation. Diamonds represent nodes that include proteins with BioB family annotation according to GO. The final BioB HMM detection threshold was achieved for the SFLD by further exploration of more strict $E$-value thresholds for edge representation, and was set to 241.6. Node and edge numbers, as well as E-value threshold, are as in Fig. 3

this chapter, for example) and edges (representing the similarity between those nodes). For the SSNs shown in this chapter, edges represent similarities scored by pairwise BLAST $E$-values (used as scores) between the source and target sequences. Using simple metrics such as these, relatively small networks are trivial and fast to produce from a simple all-against-all BLAST calculation. However, the number of edges produced depends on the similarity between all the nodes to each other, so that for comparisons of a large number of closely related sequences, the number of edges will vastly exceed the number of nodes, quickly outpacing computational resources for generating and viewing networks. As a result, some data reduction will eventually be necessary. The SFLD uses representative networks where each node represents a set of highly 


\subsection{Determining Over- and Under- represented GO Terms in a Set of Species- Diverse Proteins}

similar sequences and the edges between them represent the mean $E$-value similarity between all the sequences in the source node and all the sequences in the target node. As shown in Fig. 3, node graphical attributes (e.g. shape and colour) used to represent GO terms for the proteins shown are a powerful way to recognise relationships between sequence and functional similarities. Importantly, statistical analyses must be carried out to verify the significance of these trends, as we show below.

A common use of GO enrichment analysis is to evaluate sets of differentially expressed genes that are up- or down-regulated under certain conditions [38]. The resulting analysis identifies which GO terms are over- or under-represented within the set in question. With respect to enzyme superfamilies, the traditional implementation of enrichment analysis will not work well as there are often very many different species from different kingdoms in the dataset. However, there are several ways that we can still utilise sets of annotated proteins to evaluate the level of enrichment for GO terms.

The simplest method and least rigorous, is to take the set of proteins being evaluated, count up the number of times a single annotation occurs (including duplicate occurrences for a single enzyme, as these have different evidence sources) and up-weight for experimental (or high confidence) annotations. Then, by dividing by the number of proteins in the set, any annotation with a ratio greater than one can be considered "significant".

A more rigorous treatment assumes that for a set of closely related proteins (i.e. belonging to a family) a specific GO term is said to be over-represented when the number of proteins assigned to that term within the family of interest is enriched versus the background model as determined by a probability distribution. Thus, there are two decisions that need to be made, firstly, identifying the background model and then which probability function to use. The background model is dependent on the dataset and the question that is being asked. For example in the SFLD model, we might use the subgroup or superfamily and a random background model that gives us an idea of what annotations could occur purely by chance. The lack of high (and sometimes also medium) confidence annotations is another complication in examining enrichment of terms. If one is using IEA annotations to infer function, the assertions can quickly become circular (with inferred annotations being transferred to other proteins which in turn are used to annotate yet more proteins), leading to results which themselves are of low confidence. Similarly, if very few proteins are explicitly annotated with a high/medium confidence annotation, the measure of significance can be skewed due to low counts in the dataset. The choice of the probability function is also going to depend somewhat on what question is being asked, but the 
hypergeometric test (used for a finite universe) is common in GO analyses $[39,40]$. For more detail on enrichment analysis, see Chap. 13 [41].

\subsection{Using Semantic Significance with GO}

2.4 Use

of Orthogonal

Information

to Evaluate

GO Annotation
Instead of simply transferring annotations utilising sequence homology and BLAST scores, many tools are now available (e.g. Argot2 [42] and GraSM [43]) that utilise semantic similarity [42$46]$. Here, the idea is that in controlled vocabularies, the degree of relatedness between two entities can be assessed by comparing the semantic relationship (meanings) between their annotations. The semantic similarity measure is returned as a numerical value that quantifies the relationship between two GO terms, or two sets of terms annotating two proteins.

GO is well suited to such an approach, for example many children terms in the GO directed acyclic graph (DAG) have a similar vocabulary to their parents. The nature of the GO DAG means that a protein with a function $\mathrm{A}$ will also inherit the more generic functions that appear higher up in the DAG; this can be one or more functions, depending on the DAG. For example, an ion transmembrane transporter activity (GO:0015075) is a term similar to voltage-gated ion channel activity (GO:0005244), the latter of which is a descendent of the former, albeit separated by the ion channel activity (GO:0005216) term. Thus, the ancestry and semantic similarity lends greater weight to the confidence in the annotation.

Such similarity measures can be used instead of (or in conjunction with) sequence similarity measures. Indeed, it has been shown [47] that there is good correlation between the protein sequence similarity and the GO annotation semantic similarity for proteins in Swiss-Prot, the reviewed section of UniProtKB [17]. Consistent results, however, are often a feature not only of the branch of GO to which the annotations belong, but also the number of high confidence annotations that are being used. For a more detailed and comprehensive discussion of the various methods, see Pesquita et al. [44] and Chap. 12 [48].

In the example shown in Fig. 3, it is clear that many more nodes in the subgroup are annotated as biotin synthase by GO than match the stringent criteria set within the SFLD, which not only require a significant $E$-value (or Bit Score) to transfer annotation, but the presence of the conserved key residues. As mentioned earlier, one key advantage to using GO annotations over those of some other resources is the evidence code (and associated source of that evidence) as shown in Fig. 4. As indicated by that network, when using GO annotations, it is important to also consider the associated confidence level for the evidence used in assigning an annotation (see Table 1). In Fig. 4, only a few annotations are supported by highconfidence evidence. Alternatively, if a protein has a high confidence experimental evidence code for membership in a family of interest 
yet is not included by annotators in that family, then the definition of that family may be too strict, indicating that a more permissive gathering threshold for assignment to the family should be used.

Another way of assessing the veracity of the annotation transferred to a query protein is to examine both the annotations of the proteins that are closest to it in similarity as well as other entirely different types of information.

One example of such orthogonal information is the genomic context of the protein. It can be hypothesised that if a protein occurs in a pathway, then the other proteins involved in that pathway may be co-located within the genome [49]. This association is frequently found in prokaryotes, and to a lesser extent in plants and fungi. Genomic proximity of pathway components is infrequent in metazoans, thus genomic context as a means to function prediction is more useful for bacterial enzymes. Additionally, other genes in the same genomic neighbourhood may be relevant to understanding the function of both the protein of interest and of the associated pathway. A common genomic context for a query protein and a homologue provides further support for assignment of that function. (However, the genomic distance between pathway components in different organisms may vary for many reasons, thus the lack of similar genomic context does not suggest that the functions of a query and a similar homologue are different.)

Another type of orthogonal information that can be used can be deduced from protein domains present in a query protein and their associated annotations - what are the predicted domains present in the protein, do they all match the assigned function or are there anomalies. A good service for identifying such domains is InterProScan [50]. Further, any protein in UniProtKB will have the predicted InterPro identifiers annotated in the record (along with other predicted annotations from resources such as Pfam and CATH), along with the evidence supporting those predictions. Such sequence context can also be obtained using hidden Markov models (HMMs) [51], which is the technique used by InterPro, Pfam, Gene3D, SUPERFAMILY and the SFLD to place new sequences into families, subgroups (SFLD-specific term) and superfamilies (see Fig. 6).

\section{Challenges and Caveats}

\subsection{The Use} of Sequence Similarity Network
A significant challenge with using SSNs to help evaluate GO annotations is that SSNs are not always trivial to use without a detailed knowledge of the superfamilies that they describe. For example, choosing an appropriate threshold for drawing edges is critical to obtaining network clustering patterns useful for deeper evaluation. In Fig. 3, HydE (the blue nodes) are not currently annotated as such in GO, but are annotated instead as BioB. Thus, 


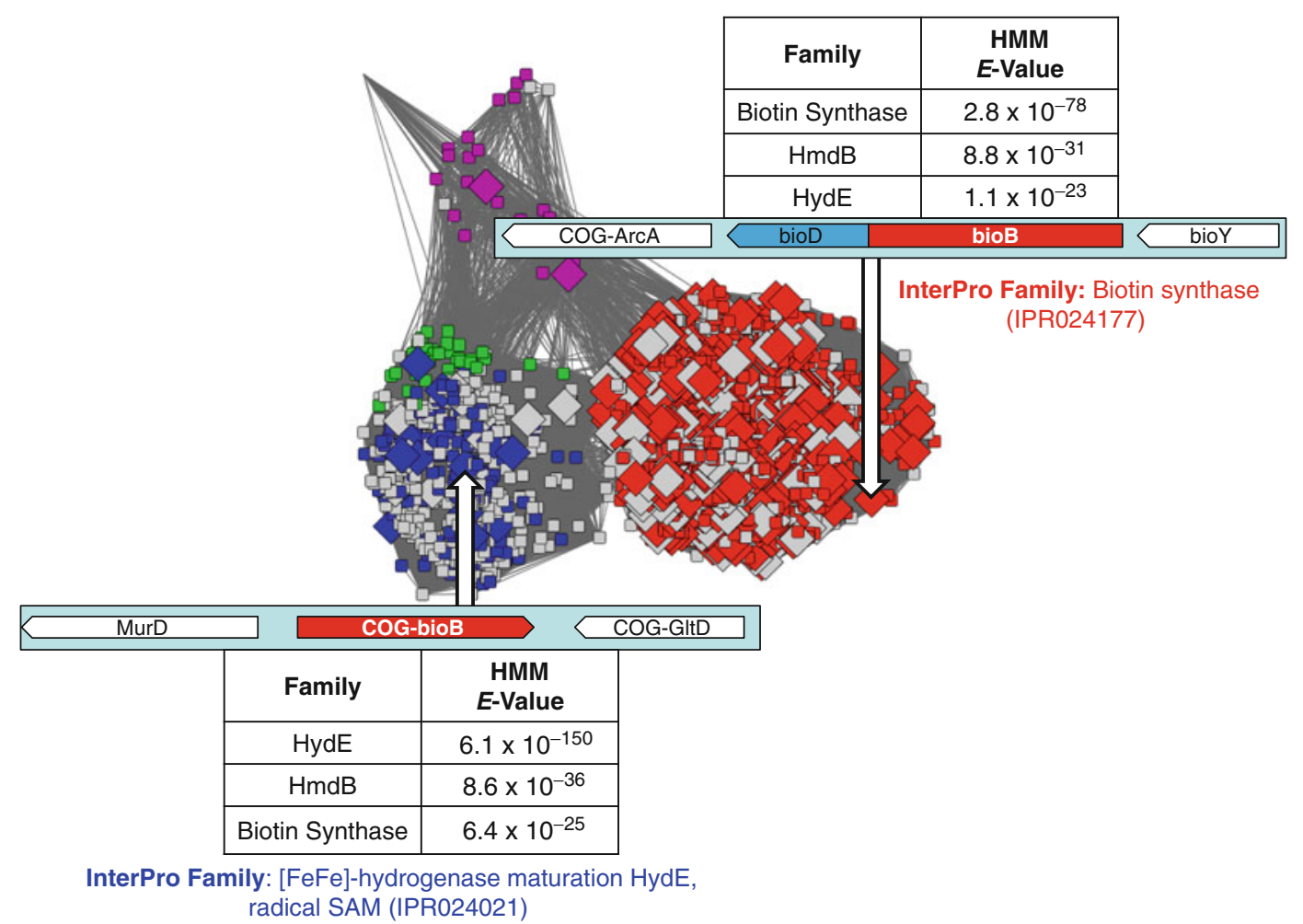

Fig. 6 Biotin synthase-like subgroup SSN showing where the biotin synthase GO annotations are shown as large diamonds. Two proteins, one from the BioB set (red nodes, top right) and one from the HydE set (blue nodes), bottom left, are shown with some associate orthogonal information: genomic context highlighted in light cyan boxes, their HMM match results for the query protein against the three top scoring families in the subgroup are shown in the tables, and family membership (according to InterProScan) shown in coloured text (blue for HydE and red for BioB). Node and edge numbers, as well as E-value threshold are as in Fig. 3. All the proteins are connected via a homologous domain (the Radical SAM domain). Thus, the observed differences in the rest of the protein mean that the functions of the proteins may also be quite different

\subsection{Annotation}

Transfer Is

Challenging Because

Evolution Is Complex the evaluation of the network becomes significantly more complex. It is also not always clear what signal is being picked up in the edge data for large networks. It is usually assumed that all the proteins in the set share a single domain, but this is often only clear when the network is examined in greater detail.

Even using the powerful tools and classifications provided by GO, interpreting protein function in many cases requires more in-depth analysis. For several reasons, it is not always easy to confidently determine that a protein is not correctly annotated. Firstly, how closely related is the enzyme to the group of interest? Perhaps we can only be relatively certain of its superfamily membership, or maybe we can assign it to a more detailed level of the functional hierarchy. If it fits into a more detailed classification level, how well does it fit? At what threshold do we begin to see false positives 
creeping into the results list? Using networks, we can also examine the closest neighbours that have differing function and ask whether there are similarities in the function (e.g. Broderick et al. [52] used sequence similarity networks to help determine the function of $\mathrm{HydE}$ ). Another complicating issue is whether a protein performs one or more promiscuous functions, albeit with a lesser efficacy.

Another important piece of evidence that can be used to support an annotation is conservation of the key residues, so it is important to assess if the protein of interest has all the relevant functional residues. Although GO includes an evidence code to handle this concept (Inferred from Key Residues, IKR), it is often not included in the electronic inference of annotations. It is important to note, however, that there are evolutionary events that may "scramble" the sequence, leaving it unclear to an initial examination whether the residues are conserved or not. A prime example is the case in which a circular permutation has occurred. Thus, it is important to look at whether there are other residues (or patterns of residues) that could perform the function of the "missing" residues. It is also possible that conservative mutations have occurred, and these may also have the ability to perform the function of the "missing" residues [53].

Another consideration with function evaluation is the occurrence of moonlighting proteins. These are proteins that are identical in terms of sequence but perform different functions in different cellular locations or species; for example argininosuccinate lyase (UniProtKB id P24058) is also a delta crystalline which serves as an eye lens protein when it is found in birds and reptiles [54]. A good source of information on moonlighting proteins is MoonProt (http://www.moonlightingproteins.org/) [55]. Such cases may arise from physiological use in many different conditions such as different subcellular localisations or regulatory pathways. The full extent of proteins that moonlight is currently not known, although to date, almost 300 cases have been reported in MoonProt. Another complicating factor for understanding the evolution of enzyme function is the apparent evolution of the same reaction specificity from different intermediate nodes in the phylogenetic tree for the superfamily, for example the $\mathrm{N}$-succinyl amino acid racemase and the muconate lactonising enzyme families in the enolase superfamily $[56,57]$.

Finally, does the protein have a multi-domain architecture and/ or is it part of a non-covalent protein-protein interaction in the cell? An example of a functional protein requiring multiple chains that are transiently coordinated in the cell is pyruvate dehydrogenase (acetyl-transferring) (EC 1.2.4.1). This protein has an active site at the interface between pyruvate dehydrogenase El component subunit alpha (UniProtKB identifier P21873) and beta (UniProtKB identifier P21874), both of which are required for activity. Thus, transfer of annotation relating to this function to an unknown (and hence evaluation of misannotation) needs to include both proteins. Similarly, a single chain with multiple domains, e.g. biotin 
biosynthesis bifunctional protein BioAB (UniProtKB identifier P53656), which contains a BioA and BioB domain, has two different functions associated with it. In this example, these two functions are distinct from one another so that annotation of this protein only with one function or the other could represent a type of misannotation (especially as a GO term is assigned to a protein, not a specific segment of its amino acid sequence).

\subsection{Plurality Vote May Not Be the Best Route}

In some cases, proteins are annotated by some type of "plurality voting". Plurality voting is simply assuming that the more annotations that come from different predictors, the more likely these are to be correct. As we have shown in this chapter (and others before us [58]), this is not always the case. An especially good example of where plurality voting fails is in the case of the lysozyme mechanism. For over 50 years, the mechanism was assumed to be dissociative, but a single experiment provided evidence of a covalent intermediate being formed in the crystal structure, calling into question the dissociate mechanism. If plurality voting were applied in ongoing annotations, the old mechanism would still be considered correct. That being said, it is more difficult to identify problems of this type if experimental evidence challenging an annotation is unavailable. In such cases, we must always look at all the available evidence to transfer function and where there are disagreements between predicted functions, a more detailed examination is needed. Only when we have resolved such issues can we have any true confidence in the plurality vote. Work by Kristensen et al. [59] provides a good example of the value of this approach. By using three-dimensional templates generated using knowledge of the evolutionarily important residues, they showed that they could identify a single most likely function in $61 \%$ of 3D structures from the Structural Genomics Initiative, and in those cases the correct function was identified with an $87 \%$ accuracy.

\section{Conclusions}

Experimentalists simply can't keep up with the huge volume of data that is being produced in today's high-throughput labs, from whole genome and population sequencing efforts to large-scale assays and structure generation. Almost all proteins will have at least one associated GO annotation, and such coverage makes GO an incredibly powerful tool, especially as it has the ability to handle all the known function information at different levels of biological granularity, has explicit tools to capture high-throughput experimental data and utilises an ontology to store the annotation and associated relationships. Although over $98 \%$ of all GO annotations are computationally inferred, with the ever-increasing state of knowledge, these annotation transfers are becoming more confident [15] as rulebased annotations gain in specificity due to more data being 
available. However, there is still a long way to go before we can simply take an IEA annotation at face value. Confidence in annotations transferred electronically has to be taken into account: How many different sources have come to the same conclusion (using different methods)? How many different proteins' functions have been determined in a single experiment? Similarly, whilst burden of evidence is a useful gauge in determining the significance of an annotation, there is also the question of when substantially different annotations were captured in GO and other resources-perhaps there has been a new experiment that calls into question the original annotation. It is also important to look at whether other, similar proteins were annotated long ago or are based on new experimental evidence. There is a wealth of data available that relates to enzymes and their functions. This ranges from the highest level of associating a protein with a superfamily (and thus giving some information as to the amino acid residues that are evolutionarily conserved), to the most detailed level of molecular function. We can use all of these data to aid us in evaluating the GO annotations for a given protein (or set of proteins), from the electronically inferred annotation for protein domain structure, to the genomic context and protein features (such as conserved residues). The more data that are available to back up (or refute) a given GO annotation, the more confident one can be in it (or not, as the case may be).

\section{Acknowledgements}

GLH and PCB acknowledge funds from National Institutes of Health and National Science Foundation (grant NIH R01 GM60595 and grant NSF DBI1356193). Open Access charges were funded by the University College London Library, the Swiss Institute of Bioinformatics, the Agassiz Foundation, and the Foundation for the University of Lausanne.

Open Access This chapter is distributed under the terms of the Creative Commons Attribution 4.0 International License (http:// creativecommons.org/licenses/by/4.0/), which permits use, duplication, adaptation, distribution and reproduction in any medium or format, as long as you give appropriate credit to the original author(s) and the source, a link is provided to the Creative Commons license and any changes made are indicated.

The images or other third party material in this chapter are included in the work's Creative Commons license, unless indicated otherwise in the credit line; if such material is not included in the work's Creative Commons license and the respective action is not permitted by statutory regulation, users will need to obtain permission from the license holder to duplicate, adapt or reproduce the material. 


\section{References}

1. Ashburner M, Ball CA, Blake JA, Botstein D, Butler H, Cherry JM, Davis AP, Dolinski K, Dwight SS, Eppig JT, Harris MA, Hill DP, Issel-Tarver L, Kasarskis A, Lewis S, Matese JC, Richardson JE, Ringwald M, Rubin GM, Sherlock G (2000) Gene ontology: tool for the unification of biology. The Gene Ontology Consortium. Nat Genet 25(1):25-29. doi:10.1038/75556

2. Nomenclature committee of the international union of biochemistry and molecular biology (NC-IUBMB), Enzyme Supplement 5 (1999). European J Biochem/FEBS 264(2):610-650

3. McDonald AG, Boyce S, Tipton KF (2009) ExplorEnz: the primary source of the IUBMB enzyme list. Nucleic Acids Res 37(Database issue):D593-D597. doi:10.1093/nar/gkn582

4. Fleischmann A, Darsow M, Degtyarenko K, Fleischmann W, Boyce S, Axelsen KB, Bairoch A, Schomburg D, Tipton KF, Apweiler R (2004) IntEnz, the integrated relational enzyme database. Nucleic Acids Res 32(Database issue):D434-D437. doi:10.1093/ nar/gkhl 19

5. Furnham N (2016) Complementary sources of protein functional information: the far side of GO. In: Dessimoz C, Škunca N (eds) The gene ontology handbook. Methods in molecular biology, vol 1446. Humana Press. Chapter 19

6. Babbitt PC (2003) Definitions of enzyme function for the structural genomics era. Curr Opin Chem Biol 7(2):230-237

7. Thomas PD (2016) The gene ontology and the meaning of biological function. In: Dessimoz C, Skunca N (eds) The gene ontology handbook. Methods in molecular biology, vol 1446. Humana Press. Chapter 2

8. Bray T, Doig AJ, Warwicker J (2009) Sequence and structural features of enzymes and their active sites by EC class. J Mol Biol 386(5):14231436. doi:10.1016/j.jmb.2008.11.057

9. Dobson PD, Doig AJ (2005) Predicting enzyme class from protein structure without alignments. J Mol Biol 345(1):187-199. doi:10.1016/j.jmb.2004.10.024

10. Cozzetto D, Jones DT (2016) Computational methods for annotation transfers from sequence. In: Dessimoz C, Škunca N (eds) The gene ontology handbook. Methods in molecular biology, vol 1446. Humana Press. Chapter 5

11. Radivojac P, Clark WT, Oron TR, Schnoes AM, Wittkop T, Sokolov A, Graim K, Funk C, Verspoor K, Ben-Hur A, Pandey G, Yunes JM, Talwalkar AS, Repo S, Souza ML, Piovesan D, Casadio R, Wang Z, Cheng J, Fang H, Gough
J, Koskinen P, Toronen P, Nokso-Koivisto J, Holm L, Cozzetto D, Buchan DW, Bryson K, Jones DT, Limaye B, Inamdar H, Datta A, Manjari SK, Joshi R, Chitale M, Kihara D, Lisewski AM, Erdin S, Venner E, Lichtarge O, Rentzsch R, Yang H, Romero AE, Bhat P, Paccanaro A, Hamp T, Kassner R, Seemayer $S$, Vicedo E, Schaefer C, Achten D, Auer F, Boehm A, Braun T, Hecht M, Heron M, Honigschmid P, Hopf TA, Kaufmann S, Kiening M, Krompass D, Landerer C, Mahlich Y, Roos M, Bjorne J, Salakoski T, Wong A, Shatkay H, Gatzmann F, Sommer I, Wass MN, Sternberg MJ, Skunca N, Supek F, Bosnjak M, Panov P, Dzeroski S, Smuc T, Kourmpetis YA, van Dijk AD, ter Braak CJ, Zhou Y, Gong Q, Dong X, Tian W, Falda M, Fontana P, Lavezzo E, Di Camillo B, Toppo S, Lan L, Djuric N, Guo Y, Vucetic S, Bairoch A, Linial M, Babbitt PC, Brenner SE, Orengo C, Rost B, Mooney SD, Friedberg I (2013) A large-scale evaluation of computational protein function prediction. Nat Methods 10(3):221-227. doi:10.1038/nmeth. 2340

12. Friedberg I, Radivojac P (2016) Communitywide evaluation of computational function prediction. In: Dessimoz C, Škunca N (eds) The gene ontology handbook. Methods in molecular biology, vol 1446. Humana Press. Chapter 10

13. Akiva E, Brown S, Almonacid DE, Barber AE 2nd, Custer AF, Hicks MA, Huang CC, Lauck F, Mashiyama ST, Meng EC, Mischel D, Morris JH, Ojha S, Schnoes AM, Stryke D, Yunes JM, Ferrin TE, Holliday GL, Babbitt PC (2014) The Structure-Function Linkage Database. Nucleic Acids Res 42(Database issue):D521-D530. doi:10.1093/nar/ gktl 130

14. Gaudet P, Škunca N, Hu JC, Dessimoz C (2016) Primer on the gene ontology. In: Dessimoz C, Škunca N (eds) The gene ontology handbook. Methods in molecular biology, vol 1446. Humana Press. Chapter 3

15. Skunca N, Altenhoff A, Dessimoz C (2012) Quality of computationally inferred gene ontology annotations. PLoS Comput Biol 8(5):e1002533. doi:10.1371/journal. pcbi. 1002533

16. Bastian FB, Chibucos MC, Gaudet P, Giglio M, Holliday GL, Huang H, Lewis SE, Niknejad A, Orchard S, Poux S, Skunca N, RobinsonRechavi M (2015) The Confidence Information Ontology: a step towards a standard for asserting confidence in annotations. Database:bav043. doi:10.1093/database/bav043 
17. UniProt C (2015) UniProt: a hub for protein information. Nucleic Acids Res 43(Database issue):D204-D212. doi:10.1093/nar/gku989

18. Hill DP, Davis AP, Richardson JE, Corradi JP, Ringwald M, Eppig JT, Blake JA (2001) Program description: strategies for biological annotation of mammalian systems: implementing gene ontologies in mouse genome informatics. Genomics 74(1):121-128. doi:10.1006/geno.2001.6513

19. Sillitoe I, Lewis TE, Cuff A, Das S, Ashford P, Dawson NL, Furnham N, Laskowski RA, Lee D, Lees JG, Lehtinen S, Studer RA, Thornton J, Orengo CA (2015) CATH: comprehensive structural and functional annotations for genome sequences. Nucleic Acids Res 43(Database issue):D376-D381. doi:10.1093/ nar/gku947

20. Lees J, Yeats C, Perkins J, Sillitoe I, Rentzsch R, Dessailly BH, Orengo C (2012) Gene3D: a domain-based resource for comparative genomics, functional annotation and protein network analysis. Nucleic Acids Res 40(Database issue):D465-D471. doi:10.1093/nar/gkrl 181

21. Fox NK, Brenner SE, Chandonia JM (2014) SCOPe: structural classification of proteins-extended, integrating SCOP and ASTRAL data and classification of new structures. Nucleic Acids Res 42(Database issue):D304D309. doi:10.1093/nar/gktl240

22. Finn RD, Bateman A, Clements J, Coggill $P$, Eberhardt RY, Eddy SR, Heger A, Hetherington K, Holm L, Mistry J, Sonnhammer EL, Tate J, Punta M (2014) Pfam: the protein families database. Nucleic Acids Res 42(Database issue):D222-D230. doi:10.1093/nar/gktl223

23. Mi H, Muruganujan A, Thomas PD (2013) PANTHER in 2013: modeling the evolution of gene function, and other gene attributes, in the context of phylogenetic trees. Nucleic Acids Res 41(Database issue):D377-D386. doi:10.1093/nar/gks1118

24. Haft DH, Selengut JD, Richter RA, Harkins D, Basu MK, Beck E (2013) TIGRFAMs and genome properties in 2013. Nucleic Acids Res 41 (Database doi:10.1093/nar/gks1234

25. Gerlt JA, Babbitt PC (2001) Divergent evolution of enzymatic function: mechanistically diverse superfamilies and functionally distinct suprafamilies. Annu Rev Biochem 70:209246. doi:10.1146/annurev.biochem.70.1.209

26. Camacho C, Coulouris G, Avagyan V, Ma N, Papadopoulos J, Bealer K, Madden TL (2009) BLAST+: architecture and applications. BMC Bioinformatics 10:421. doi:10.1186/14712105-10-421
27. Finn RD, Clements J, Eddy SR (2011) HMMER web server: interactive sequence similarity searching. Nucleic Acids Res 39(Web Server issue):W29-W37. doi:10.1093/nar/ gkr367

28. Brown SD, Babbitt PC (2014) New insights about enzyme evolution from large scale studies of sequence and structure relationships. J Biol Chem 289(44):30221-30228. doi:10.1074/ jbc.Rl14.569350

29. Schnoes AM, Brown SD, Dodevski I, Babbitt PC (2009) Annotation error in public databases: misannotation of molecular function in enzyme superfamilies. PLoS Comput Biol 5(12):e1000605. doi:10.1371/journal. pcbi. 1000605

30. Pieper U, Chiang R, Seffernick JJ, Brown SD, Glasner ME, Kelly L, Eswar N, Sauder JM, Bonanno JB, Swaminathan S, Burley SK, Zheng X, Chance MR, Almo SC, Gerlt JA, Raushel FM, Jacobson MP, Babbitt PC, Sali A (2009) Target selection and annotation for the structural genomics of the amidohydrolase and enolase superfamilies. J Struct Funct Genom 10(2):107-125. doi:10.1007/ s10969-008-9056-5

31. Gerlt JA, Babbitt PC, Jacobson MP, Almo SC (2012) Divergent evolution in enolase superfamily: strategies for assigning functions. J Biol Chem 287(1):29-34. doi:10.1074/jbc. Rl11.240945

32. Mashiyama ST, Malabanan MM, Akiva E, Bhosle R, Branch MC, Hillerich B, Jagessar K, Kim J, Patskovsky Y, Seidel RD, Stead M, Toro $\mathrm{R}$, Vetting MW, Almo SC, Armstrong RN, Babbitt PC (2014) Large-scale determination of sequence, structure, and function relationships in cytosolic glutathione transferases across the biosphere. PLoS Biol 12(4):el001843. doi:10.1371/journal.pbio.1001843

33. Rentzsch R, Orengo CA (2013) Protein function prediction using domain families. BMC Bioinformatics 14(Suppl 3):S5. doi:10.1186/1471-2105-14-S3-S5

34. Atkinson HJ, Morris JH, Ferrin TE, Babbitt PC (2009) Using sequence similarity networks for visualization of relationships across diverse protein superfamilies. PLoS One 4(2):e4345. doi:10.1371/journal.pone.0004345

35. Barber AE II, Babbitt PC (2012) Pythoscape: a framework for generation of large protein similarity networks. Bioinformatics. doi:10.1093/ bioinformatics/bts532

36. Gerlt JA, Bouvier JT, Davidson DB, Imker HJ, Sadkhin B, Slater DR, Whalen KL (2015) Enzyme Function Initiative-Enzyme Similarity Tool (EFI-EST): a web tool for generating protein sequence similarity networks. Biochim 
Biophys Acta 1854(8):1019-1037. doi:10.1016/j.bbapap.2015.04.015

37. Mitchell A, Chang HY, Daugherty L, Fraser M, Hunter S, Lopez R, McAnulla C, McMenamin C, Nuka G, Pesseat S, SangradorVegas A, Scheremetjew M, Rato C, Yong SY, Bateman A, Punta M, Attwood TK, Sigrist CJ, Redaschi N, Rivoire C, Xenarios I, Kahn D, Guyot D, Bork P, Letunic I, Gough J, Oates $\mathrm{M}$, Haft $\mathrm{D}$, Huang $\mathrm{H}$, Natale $\mathrm{DA}$, Wu $\mathrm{CH}$, Orengo C, Sillitoe I, Mi H, Thomas PD, Finn $\mathrm{RD}$ (2014) The InterPro protein families database: the classification resource after 15 years. Nucleic Acids Res. doi:10.1093/nar/gkul243

38. Webber C (2011) Functional enrichment analysis with structural variants: pitfalls and strategies. Cytogenet Genome Res 135(3-4):277-285. doi:10.1159/000331670

39. Thomas PD, Wood V, Mungall CJ, Lewis SE, Blake JA, Gene Ontology C (2012) On the use of gene ontology annotations to assess functional similarity among orthologs and paralogs: a short report. PLoS Comput Biol $8(2)$ :e1002386. pcbi. 1002386

40. Cao J, Zhang S (2014) A Bayesian extension of the hypergeometric test for functional enrichment analysis. Biometrics 70(1):84-94. doi:10.1111/biom.12122

41. Bauer S (2016) Gene-category analysis. In: Dessimoz C, Škunca N (eds) The gene ontology handbook. Methods in molecular biology, vol 1446. Humana Press. Chapter 13

42. Falda M, Toppo S, Pescarolo A, Lavezzo E, Di Camillo B, Facchinetti A, Cilia E, Velasco R, Fontana P (2012) Argot2: a large scale function prediction tool relying on semantic similarity of weighted Gene Ontology terms. BMC Bioinformatics 13(Suppl 4):S14. doi:10.1186/1471-2105-13-S4-S14

43. Couto FM, Silva MJ, Coutinho PM (2007) Measuring semantic similarity between Gene Ontology terms. Data Knowl Eng 61(1):137152. doi:10.1016/j.datak.2006.05.003

44. Pesquita C, Faria D, Falcao AO, Lord P, Couto FM (2009) Semantic similarity in biomedical ontologies. PLoS Comput Biol 5(7):e1000443. doi:10.1371/journal.pcbi.1000443

45. Benabderrahmane S, Smail-Tabbone M, Poch O, Napoli A, Devignes MD (2010) IntelliGO: a new vector-based semantic similarity measure including annotation origin. BMC Bioinformatics 11:588. doi:10.1186/1471-2105-11-588

46. Wu X, Pang E, Lin K, Pei ZM (2013) Improving the measurement of semantic similarity between gene ontology terms and gene products: insights from an edge- and IC-based hybrid method. PLoS One 8(5):e66745. doi:10.1371/journal.pone.0066745

47. Apweiler R, Bairoch A, Wu CH, Barker WC, Boeckmann B, Ferro S, Gasteiger E, Huang H, Lopez R, Magrane M, Martin MJ, Natale DA, O'Donovan C, Redaschi N, Yeh LS (2004) UniProt: the Universal Protein knowledgebase. Nucleic Acids Res 32(Database issue):D115-D119. doi:10.1093/nar/gkhl31

48. Pesquita C (2016) Semantic similarity in the gene ontology. In: Dessimoz C, Škunca N (eds) The gene ontology handbook. Methods in molecular biology, vol 1446. Humana Press. Chapter 12

49. Huynen M, Snel B, Lathe W, Bork P (2000) Exploitation of gene context. Curr Opin Struct Biol 10(3):366-370

50. Li W, Cowley A, Uludag M, Gur T, McWilliam H, Squizzato S, Park YM, Buso N, Lopez R (2015) The EMBL-EBI bioinformatics web and programmatic tools framework. Nucleic Acids Res. doi:10.1093/nar/gkv279

51. Meng X, Ji Y (2013) Modern computational techniques for the HMMER sequence analysis. ISRN Bioinformatics 2013:252183. doi: $10.1155 / 2013 / 252183$

52. Betz JN, Boswell NW, Fugate CJ, Holliday GL, Akiva E, Scott AG, Babbitt PC, Peters JW, Shepard EM, Broderick JB (2015) [FeFe]hydrogenase maturation: insights into the role HydE plays in dithiomethylamine biosynthesis. Biochemistry 54(9):1807-1818. doi:10.1021/ bi501205e

53. Wellner A, Raitses Gurevich M, Tawfik DS (2013) Mechanisms of protein sequence divergence and incompatibility. PLoS Genet 9(7):e1003665. doi:10.1371/journal. pgen. 1003665

54. Sampaleanu LM, Yu B, Howell PL (2002) Mutational analysis of duck delta 2 crystallin and the structure of an inactive mutant with bound substrate provide insight into the enzymatic mechanism of argininosuccinate lyase. J Biol Chem 277(6):4166-4175. doi:10.1074/ jbc.M107465200

55. Mani M, Chen C, Amblee V, Liu H, Mathur T, Zwicke G, Zabad S, Patel B, Thakkar J, Jeffery CJ (2015) MoonProt: a database for proteins that are known to moonlight. Nucleic Acids Res 43(Database issue):D277-D282. doi:10.1093/nar/gku954

56. Song L, Kalyanaraman C, Fedorov AA, Fedorov EV, Glasner ME, Brown S, Imker HJ, Babbitt PC, Almo SC, Jacobson MP, Gerlt JA (2007) Prediction and assignment of function 
for a divergent $\mathrm{N}$-succinyl amino acid racemase. Nat Chem Biol 3(8):486-491. doi:10.1038/nchembio.2007.11

57. Sakai A, Fedorov AA, Fedorov EV, Schnoes AM, Glasner ME, Brown S, Rutter ME, Bain K, Chang S, Gheyi T, Sauder JM, Burley SK, Babbitt PC, Almo SC, Gerlt JA (2009) Evolution of enzymatic activities in the enolase superfamily: stereochemically distinct mechanisms in two families of cis, cis-muconate lac- tonizing enzymes. Biochemistry 48(7):1445-1453. doi:10.1021/bi802277h

58. Brenner SE (1999) Errors in genome annotation. Trends Genet 15(4):132-133

59. Kristensen DM, Ward RM, Lisewski AM, Erdin $S$, Chen BY, Fofanov VY, Kimmel M, Kavraki LE, Lichtarge O (2008) Prediction of enzyme function based on 3D templates of evolutionarily important amino acids. BMC Bioinformatics 9:17. doi:10.1186/1471-2105-9-17 